\title{
Fetal Heart Rate Monitoring
}

\section{Kazuo Maeda*}

Department of Obstetrics and Gynecology, Tottori University Medical School, Yonago, Japan

\section{Introduction}

Fetal Heart Rate (FHR) monitoring is indispensable in modern management of the fetus mainly in intrapartum stage, but also during high-risk pregnancy, to reduce fetal or neonatal demise, to prevent severe neonatal asphyxia and sometimes it is requested to prevent infantile Cerebral Palsy (CP) due to fetal hypoxia.

In old times, fetal heart sound was auscultated by a stethoscope or ultrasonic Doppler fetal heart detector, but they were replaced by the continuous chart record of FHR with FHR meter, which has been traced simultaneously with the uterine contraction (cardiotacograph, CTG) or recently with fetal movement signals (actocardiograph).

FHR is traced by a FHR meter with the instantaneous FHR meter internally using Fetal Scalp Lead Electrocardiogram (FECG) (Electronic Fetal Monitoring, EFM) or externally detected fetal heart sound or ultrasonic Doppler fetal heart beat signals (External FHR monitoring). Uterine contraction was recorded by intrauterine pressure using intrauterine catheter in the EFM, or by external took dynamometer used on maternal abdominal surface. The FHR should be monitored in every state of pregnant women, not only in the labor, but any time in the late half of pregnancy, i.e. not only in the labor but before the onset of labor contraction, because fetal hypoxia and asphyxia should be detected not only in the labor but also during pregnancy. The application of fetal skin penetrating needle electrode is contraindicated in maternal infection of pathological virus or bacteria to prevent vertical fetal infection [1]. Thus, the use of EFM recorded with needle electrode is limited in non-infectious cases. The FHR diagnosis was the evaluation of discrete FHR changes in FHR pattern diagnosis including bradycardia, tachycardia. The loss of irregularity, late deceleration or severe variable deceleration [2], to detect imminent fetal damages which are prevented by artificial rapid delivery, including Cesarean section in the 1st stage of labor, forceps or vacuum extraction in the 2nd stage. However, late deceleration was not always abnormal (Table 1) [3], and the pattern diagnosis did not contain comprehensive fetal evaluation.

From these points of view, external FHR monitoring was more suitable to monitor any case in the late half of pregnancy through the birth than internal direct FHR method. Since Doppler fetal heart detector

\begin{tabular}{|l|l|l|l|}
\hline \multicolumn{2}{|l|}{ FHR changes } & $\begin{array}{l}\text { Low Apgar score } \\
<7 \text { (\%) }\end{array}$ & Evaluation score \\
\hline Baseline FHR deceleration & $\begin{array}{l}<110 \text { or }> \\
180 \mathrm{bpm}\end{array}$ & 71 & 3 \\
\hline Nadir heart rate (bpm) & $<100$ & 27 & 2 \\
\hline Amplitude (bpm) & $>50$ & 50 & 2 \\
\hline Duration (sec) & $>60$ & 71 & 3 \\
\hline Recovery time (sec) & $>40$ & 63 & 3 \\
\hline Lag time (sec) & $>40$ & 71 & 3 \\
\hline Loss of acceleration & & 45 & 2 \\
\hline Loss of variability & & 100 & 4 \\
\hline
\end{tabular}

Table 1: Evaluation of discrete FHR changes.

Comprehensive FHR score is abnormal when FHR score is $10-19$, and highly abnormal when it is 20 or more. It is the summary of each evaluation scores of discrete FHR changes, of which abnormality is shown in the ratio of low Apgar. had excellent fetal heart beat detection in the labor, ultrasonic Doppler FHR monitor was strongly hoped, but there were two problems in the Doppler ultrasound. The safety of medical ultrasound was concerned even in the FHR monitoring, but Tottori University group found that ultrasound had no bioeffect if ultrasound intensity was weak [4]. The FHR baseline disturbance in the use of ultrasound Doppler was solved by the autocorrelation FHR meter [5]. Thus the external ultrasound Doppler FHR monitor is widely utilized in the FHR monitoring, which enables FHR monitoring in all pregnant women without limitation. In addition, the ultrasound detects fetal movement directly at fetal chest, and simultaneous fetal movement is recorded on the chart in the actocardiogram (ACG), using the same ultrasound to detect fetal heart beat without increasing ultrasound intensity. The ACG solved various diagnostic Problems by the analysis of fetal movements [6].

A new trend of FHR monitoring is the use of multiple electrodes on thesurface of maternal abdomen to detect fetal ECG spikes deleting maternal ECG using instantaneous FHR meter and short term variability (STV) [7]. Also, the fetal magnetocardiography (FMCG) detects fetal QRS without maternal signal, and applied to FHR monitoring [8]. Uterine contraction is recorded processing the electrohysterogram, the electric activity of uterine muscle.

Although the fetal monitoring improved fetal outcome in the reduction offetal and neonatal deaths reducing perinatal mortality, by reducing neonatal asphyxia, and also by reducing cerebral palsy in Japan $[9,10]$, the watching and evaluating fetal state through the fetal delivery was a troublesome work for obstetric staffs. Also, the parallel fetal monitoring is difficult in the hospital of large number of births. An automatic computerized fetal monitoring is introduced into the obstetrics to solve monitoring problems $[11,12]$.

\section{References}

1. Intrapartum surveillance: recommendations on current practice and overview of new developments. FIGO Study Group on the Assessment of New Technology. International Federation of Gynecology and Obstetrics (1995). Int J GynecolObstet 49: 213-221.

2. Hon EH (1968) An Atlas of Fetal Heart Rate Patterns. Harty Press, New Haven, 305.

3. Maeda K, Kimura S, Nakano H (1969) Pathophysiology of Fetus. Fukuoka Printing, Fukuoka, Japan.

4. Maeda K, Murao F, Yoshiga T, Yamauchi C, Tsuzaki T (1986) E Experimental Studies on the Suppression of Cultured Cell Growth Curves After Irradiation with CW and Pulsed Ultrasound. IEEE Trans UFFC 33: 186-193.

*Corresponding author: Kazuo Maeda, Department of Obstetrics and Gynecology, Tottori University Medical School, Yonago, Japan, E-mail: maedak@mocha.ocn.ne.jp

Received October 14, 2013; Accepted October 15, 2013; Published October 19 2013

Citation: Maeda K (2014) Fetal Heart Rate Monitoring. J Health Med Informat 5 e112. doi:10.4172/2157-7420.1000e112

Copyright: (c) 2014 Maeda K. This is an open-access article distributed under the terms of the Creative Commons Attribution License, which permits unrestricted use, distribution, and reproduction in any medium, provided the original author and source are credited. 
5. Takeuchi Y, Hogaki M (1977) Autocorrelation method for fetal heart rate management from ultrasonic Doppler fetal signal. Ultrasound in Medicine Vol 3B, Plenum Press New York, 1327-1332

6. Maeda K (2013) Actocardiogram. Tokyo, Medical View.

7. Kimura Y, Sato N, Sugawara J, Clarissa V, Teturo H, et al. (2012) Recent advances in fetal electrocardiography. The Open Medical Devices Journal 4 7-12.

8. Fukushima A, Matsumoto A, Terata M, TomonobuKanasugi, Kenji Nakai, et al. (2012) Prenatal Diagnosis of fetal arrhythmia and assessment of automatic nervous system activity by fetal magnetocardiography. The Open Medical Devices Journal 4: 13-21.

9. Takeshita K, Ando Y,Ohtani K, Takashima S (1989) Cerebral palsy in
Tottori, Japan. Benefits and risks of progress in perinatal medicine. Neuroepidemiology4: 184-192.

10. Tsuzaki T, Sekijima A, Morishita K, Takeuchi Y, Mizuta M, et al. (1990) The survey on the perinatal variables and the incidence of cerebral palsy for 12 years before and after the application of the fetal monitoring systems. Nihon SankaFujinkaGakkaiZasshi42: 99-105.

11. Maeda K, Tatsumura M, Nagasawa T, et al. Computer-aided fetal heart ate analysis and fetal-distress diagnosis during labor and pregnancy utilizing external technique in fetal monitoring. In DAB Lindburg, S Kawahara eds. MEDINFO 80, 1980, North Holland, Amsterdam, 1214-1218.

12. Maeda K, Noguchi Y, Matsumoto F, Nagasawa T (2006) Quantitative fetal heart rate evaluation without pattern classification: FHR score and artificial neura network analysis. Network 21: 127-141. 